five dissections out of 30 (Brash, 1955). It is of interest to note that high radial fractures occurred in two of the three cases described. Reduction of such fractures is easier in supination, and stripping of the flexor pollicis longus is less necessary for exposure. Thus conditions are eminently favourable for a traction injury of the nerve. In Case 3 the radial fracture was not high and the paralysis has been permanent. In this case the cause may be different.

The cases in this series differ from those with closed forearm fractures described by Warren (1963), in which the flexor digitorum profundus to index was paralysed in addition to the long thumb flexor. This indicates that the nerve trunk was involved, since the anterior interosseous nerve constantly supplies the index segment of the profundus muscle (Seddon, 1954). The pronator quadratus will be paralysed in both types of injury, but there are no clinical means of detecting this.

\section{Summary}

Four cases of nerve lesions after plating of forearm bones are described. The probable cause of such lesions is discussed. The prognosis generally seems to be fairly good and recovery can be expected in most cases.

It is a pleasure to acknowledge the help and advice of Mr. W. Sillar and Professor R. Barnes in the preparation of this paper. The illustrations are the work of Mr. J. Loudon Brown, of the Southern General Hospital, Glasgow.

\section{REFERENCES}

Brash, J. C. (1955). Neuro-vascular Hila of Limb Muscles, p. 18. Livingstone, Edinburgh.

Burwell, H. N., and Charnley, A. D. (1964). 7. Bone ft Surg., 46B, 404. Seddon, H. J. (1954). Spec. Rep. Ser. med. Res. Coun. (Lond.), No. 282, p. 3.

Warren, J. D. (1963). f. Bone ft Surg., 45B, 511.

\title{
Acute Non-specific Pericarditis. A Description of Nineteen Cases
}

\author{
ANTHONY MARTIN,* M.B., B.S.
}

Brit. med. F., 1966, 2, 279-281

Acute non-specific pericarditis has been a recognized condition for many years. The first case was described by Hodges in 1854. It was not, however, until 1942 that Barnes and Burchell reported 14 cases of acute non-specific pericarditis simulating myocardial infarction. Since then numerous cases have been reported, mostly in the American literature, but only one large series (14 cases) has been described in the British literature (Swan, 1960). The present paper reports 19 cases that have been seen in one peripheral general hospital of 550 bedsmost of them within one year.

The clinical picture of acute non-specific pericarditis is familiar and classically presents with the sudden onset of dull aching retrosternal pain, which may be severe and often follows a recent infection of the upper respiratory tract. The pain may radiate to the shoulders, neck, back, or down the arms. It is often made worse by deep breathing or from lying on the back or left side. A pericardial friction rub may be heard, and the E.C.G. shows elevation of the S-T segments over a varying number of leads. There is usually a polymorph leucocytosis. The illness is characteristically benign and the patient usually improves symptomatically within a week or 10 days and is completely well in six to eight weeks. In a few days the cardiographic appearances alter; the S-T segments return towards the iso-electric line, and after a week the $T$-waves become inverted. There is no reciprocal depression of the S-T segments, and the QRS complexes are not disturbed unless there is a pericardial effusion. The $T$-waves revert to normal at any time between a few days and several weeks.

\section{Clinical Features}

The following Table gives the clinical features, age incidence, and sex ratio of the cases in the series.

Premonitory Chest Pain.-This occurred in six patientsin four cases one week, in one case three weeks, and in another case four weeks before their admission to hospital. These attacks of pain were identical in character and distribution with the attacks which necessitated their admission to hospital, but less severe and of shorter duration, usually four to six

* Medical Registrar, Orpington Hospital, Orpington, Kent. hours. After these brief attacks all the patients were quite well, and in only one was there a history of upper respiratory infection.

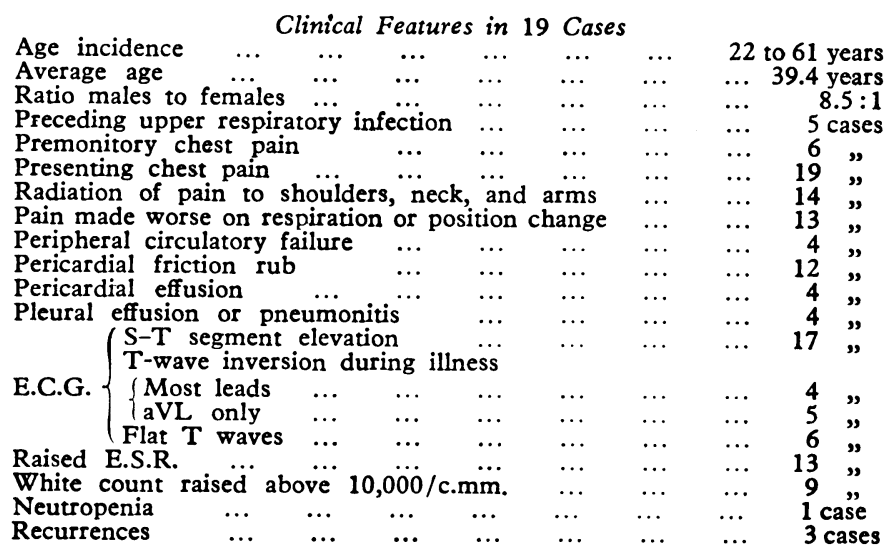

Presenting Chest Pain.-All patients in this series presented with pain in the chest. This was generally fairly severe and persisted for two to three days; it was their major complaint. In four cases it was extremely severe, these patients being in a shocked state on admission-pale, sweating, and with marked hypotension; in three a myocardial infarction was initially suspected, and one was treated with anticoagulants for 24 hours. Chest pain in all cases was retrosternal, and dull and aching in character. In 14 cases the pain radiated to either one or other shoulder, to both in some, and in one case down the arms. In three patients the pain radiated up into the neck; in 13 it was markedly worsened by deep breathing and in five by lying on the left side.

Pericardial Friction Rub.-A pericardial rub was heard at some stage of the illness in 12 cases. In all but one it was heard on admission. It was typically loud and widespread over the praecordium, and was noted to disappear for varying intervals. In one case the pericardial rub persisted for 16 days and in another for seven days. In the other 10 cases the rub lasted for two to four days. No correlation was noted between the duration and severity of the illness and the presence or duration of the pericardial rub. 
Electrocardiographic Appearances.-Seventeen of the cases showed an inital S-T segment elevation of more than $2 \mathrm{~mm}$. involving most of the chest leads and usually the unipolar limb leads as well. None had reciprocal $S-T$ depression in any lead. The S-T segment elevation initially had a scaphoid depression in it, and after three days the $S-T$ segments became rectilinear but were still elevated. One patient presented with $T$-wave inversion in all leads, but he had been ill for a week before admission. Four patients developed $T$-wave inversion in most leads after about a week. A further five cases developed $\mathrm{T}$-wave inversion in only one lead (aVL) even though they had previously had widespread S-T elevation at the onset of the illness. The $T$-wave inversion usually reverted to normal after a fortnight, but in one case it remained inverted for a month. Flat T-waves in several leads, and in three cases in all leads, developed in six cases. All but one of these cases later developed $\mathrm{T}$-wave inversion, and thus there were five in which no $\mathrm{T}$-wave disturbance occurred at all throughout the illness. In no case was there any disturbance of the QRS complexes nor any degree of conduction delay. E.C.G. abnormalities persisted on average two to three weeks, but varied from eight days to six weeks. There was no relation between the degree and rate of development of E.C.G. abnormalities and the severity of the clinical illness.

Haematological Findings.- Thirteen patients initially had an elevated sedimentation rate; these rates varied widely, but generally were between 20 and $30 \mathrm{~mm}$. $/ \mathrm{hr}$. (Westergren). One case had an E.S.R. of $100 \mathrm{~mm} . / \mathrm{hr}$. and another of $83 \mathrm{~mm} . / \mathrm{hr}$. Nine cases had a white-cell count of about 10,000/c.mm. In eight of these there was predominantly a polymorph leucocytosis. One had a marked eosinophilia which persisted for 10 days, though his sedimentation rate was normal; no other cause of eosinophilia was found. Neutropenia occurred in one patient. One patient had a white-cell count of 10,000, of which $12 \%$ were monocytes. His E.S.R. was $83 \mathrm{~mm} . / \mathrm{hr}$., and the Paul-Bunnell test was negative. Abnormal mononuclear cells were not observed in any patient. Only three patients had a normal white blood count and sedimentation rate.

Other Investigations.-In most cases an intensive search was made for a possible aetiological factor. None had a previous history of rheumatic fever. The serum transaminase level was estimated on three consecutive days in 14 patients and was normal on each occasion. The antistreptolysin $O$ titre was estimated in 11 patients and in all was within normal limits. Virus studies, stool culture, and serum agglutination investigations were carried out in 12 patients and were negative in 11 . Coxsackie $B_{4}$ virus was grown from the faeces of one patient but virus agglutination reactions were negative. This patient developed glandular fever one month after his discharge from hospital.

\section{Discussion}

Only five out of 19 patients in the present series had a previous history of upper respiratory infection. This is a smaller proportion than in previously reported series. Scherl (1956) noted that $43 \%$ of his cases had had a previous upper respiratory infection, and most other workers found that the figures were usually between 50 and $80 \%$ (Swan, 1960 ; Gilley, McCord, and Taguchi, 1951 ; Krook, 1954).

The incidence of chest pain, its character and distribution, was classical in the present series. Four cases, however, presented with shock, and in one this led to a confident initial diagnosis of myocardial infarction. It cannot be stressed too strongly how important it is to distinguish acute non-specific pericarditis from myocardial infarction. Anticoagulants may be extremely dangerous in acute non-specific pericarditis, and some cases of death from cardiac tamponade due to haemopericardium have been reported (Brit. med. F., 1965). There is no doubt that haemopericardium may be worsened, if not precipitated, by anticoagulants. Though the prognosis in acute non-specific pericarditis is usually extremely good, 11 fatal cases have been reported in the world literature. Krook (1954) also reported two cases that developed constrictive pericarditis. In the present series all patients made a uniformly good recovery. The chest pain in acute non-specific pericarditis is usually less severe than in myocardial infarction. It may radiate to the shoulders, neck, or down the arms, and there may be numbness of the fingers. In this series 14 cases showed one or other of these symptoms-a much higher incidence than the $30 \%$ found by Scherl (1956). Unlike myocardial infarction, the pain of acute non-specific pericarditis is often made worse by certain movements, such as lying on the left side or back, and may also be aggravated by deep breathing ; 13 cases presented with one or other of these symptoms. The pain is thought to be referred from the left lateral portion of the parietal pericardium to the fifth and sixth intercostal nerves (Rosenow and Cross, 1951).

Although 12 of the 19 cases had a pericardial friction rub, which is in keeping with the findings in other reports, its duration was rather less than generally recorded. Levy and Patterson (1950) reported that the average duration of the pericardial rub in acute non-specific pericarditis was nine days. The incidence of pericardial effusion $(20 \%)$ was noticeably lower in the present series than in Scherl's cases $(50 \%)$ and in other reports, where it was noted in 33 to $68 \%$ (Parker and Cooper, 1951; Rosenow and Cross, 1951 ; Krook, 1954). Similarly, pleural effusion and pneumonitis occurred rather less often than in previous series (Parker and Cooper, 1951; Krook, 1954 ; Scherl, 1956).

The present report emphasizes the variability of electrocardiographic changes in acute non-specific pericarditis, the only consistent factor being the initial S-T segment elevation, which may occur in a variable number of leads. The $\mathrm{T}$-wave disturbances in this series were found to be the exception rather than the rule. Indeed, nine cases had no $\mathrm{T}$-wave disturbances at all. Five had $\mathrm{T}$-wave inversion occurring in only one lead (aVL) which would appear to be significant, and only four cases had $\mathrm{T}$-wave inversion in more than one lead. It is interesting that three of the four cases with pericardial effusion also showed $\mathrm{T}$-wave inversion in most leads. It may be that $\mathrm{T}$-wave inversion is produced only in cases with pericardial effusion. This would account for both the low incidence of $T$-wave inversion found in the present series and the low incidence of pericardial effusion. The $T$-wave inversion in acute non-specific pericarditis may fluctuate rapidly, and this may possibly be missed unless frequent E.C.G. tracings are made.

The illness may recur even after a few weeks, and possibly after many months (Logue and Wendkos, 1948 ; Krook, 1954 ; Scherl, 1956). In this series no patient relapsed within a few months of the illness; three had had other attacks separated by one or two years. Six patients, however, described attacks of chest pain of mild degree and short duration within a month prior to their admission; and though it is possible that they had had previous subclinical acute non-specific pericarditis it was never severe enough for them to seek medical attention. These features would seem to be a useful diagnostic aid in investigating the clinical history.

Unfortunately, it has again been impossible to establish any cause for acute non-specific pericarditis in these cases. Many authors feel that it may be due to a virus infection, and certainly cases of virus pericarditis have been ascribed to Coxsackie virus (Fletcher and Brennan, 1957), lymphogranuloma venereum (Sheldon, Wall, Slade, and Heyman, 1948), Bornholm's disease, and infectious mononucleosis (Gardner, 1959). Alternatively, acute non-specific pericarditis may be due to an allergic phenomenon, and a similar clinical picture occurs in the postmyocardial infarction syndrome (Dressler, 1959 ; Davidson, Oliver, and Robertson, 1961) and the post-commissurotomy 
syndrome ; it has also been described in Reiter's disease (C'sonka and Oates, 1957). It is important to exclude other common causes of pericarditis such as rheumatic fever, myocardial infarction, collagen disorders, and neoplastic disease.

All patients in the present series were given purely supportive therapy with analgesics and bed rest. No antibiotics or corticosteroids were used, and all patients made relatively rapid and uneventful recoveries.

\section{Summary}

Nineeteen cases of acute non-specific pericarditis are presented, the majority of which were seen in one hospital within one year. It would appear that the illness is much more common than previous reports would indicate. The importance of differentiating acute non-specific pericarditis from myocardial infarction is discussed. Pericarditis can be diagnosed only when either a pericardial friction rub is heard or the electrocardiograph shows $S-T$ segment elevation without pathological Q-waves or S-T segment depression in the reciprocal leads. Premonitory chest pain is an important finding in acute nonspecific pericarditis. The blood sedimentation rate is usually accelerated, but a neutrophilia in the peripheral blood film occurs in less than half the cases. All the present cases made a uniformly good recovery.

I am indebted to Dr. Lawson McDonald for reading this paper and for his helpful criticism. I wish to thank Dr. Neville Southwell and Dr. John D. Williams for permission to publish details of cases admitted under their care, and for much helpful advice and encouragement.

REFERENCES

Barnes, A. R., and Burchell, H. B. (1942). Amer. Heart f., 23, 247 Brit. med. F., 1965, 2, 60.

Csonka, G. W., and Oates, J. K. (1957). Brit. med. F., 1, 866

Davidson, C., Oliver, M. F., and Robertson, R. F. (1961). Ibid., 2, 535. Dressler, W.' (1959). Arch., intern. Med., 103, 28 .

Fletcher, E., and Brennan, C. F. (1957). Lancet, 1, 913.

Fletcher, E., and Brennan, C. F. (1957). Lancet, 1, 913.

Gardner, C. C. (1959). Amer. F. med. Sci., 237, 352. 249.

Krook, H. (1954). Acta med. scand., 148, 201.

Levy, R. L., and Patterson, M. C. (1950). Amer. 7. Med., 8, 34.

Logue, R. B., and Wendkos, M. H. (1948). Amer. Heart 7., 36, 587.

Parker, R. C., and Cooper, H. R. (1951). F. Amer. med. Ass., 147, 835. Rosenow, O. F., and Cross, C. J. (1951). Arch. intern. Med., 87, 795.

Scherl, N. D. (1956). 7. Mt Sinai Hosp., 23, 293.

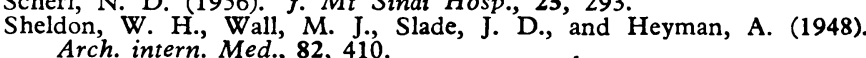

Swan, W. G. A. (1960). Brit. Heart 7., 22, 651 .

\section{Medical Memoranda}

\section{Generalized Peritonitis due to Spontaneous Intraperitoneal Rupture of Pyonephrosis}

Brit. med. F., 1966, 2, 281-282

Among the causes of generalized peritonitis an intraperitoneal rupture of pyonephrosis has been mentioned in the textbooks as an exceptionally rare occurrence. Only a few cases have been reported ; an account is now presented of the successful surgical treatment of a patient with this condition.

\section{CASE RePort}

A married woman aged 33 was seen in the outpatient clinic on 3 March 1965 complaining of a constant ache in the left loin, occasionally radiating round to the left groin, for the previous six weeks, accompanied by loss of appetite and loss of $10 \mathrm{lb} .(4.5 \mathrm{~kg}$.) in weight. She had vomited for two days prior to her visit. There were no associated micturition symptoms. A symptom-free urinary infection towards the end of pregnancy in September 1964 had been treated with chemotherapy. Examination revealed a tall, thin quiet, and miserable patient. The right kidney was easily palpable. There was tenderness in the left loin. Examination of urine showed: R.B.C. ++++ ; pus cells ++ ; profuse growth of coliform sensitive to ampicillin. Radiography showed a large staghorn calculus occupying the left renal pelvis and upper caliceal complex, a separate calculus in the left lateral upper calix, and numerous further calculi in the left lower calices, with apparently some calcification in the renal substance of the left lower pole. There was no excretion in the left kidney. Compensatory hypertrophy of the right kidney was apparent.

On the night of 15 March she was admitted as an emergency case, with a complaint of sudden severe central abdominal pain of two hours' duration, which later had shifted to the left iliac fossa. The pain was constant, was made worse by movement, and was maximal on the left side. She also had had rigor on the morning of admission. Urinary symptoms were still absent.
On admission she was thin and pale, with sunken eyes. The tongue was furred and dry. Temperature was $99.4^{\circ} \mathrm{F}$. $\left(37.4^{\circ} \mathrm{C}\right.$.), pulse 120 , and B.P. $110 / 60$. The abdomen was generally tendermaximally in the left lower quadrant-and rigid with rebound tenderness. Bowel sounds were absent. Rectal examination revealed tenderness and fullness in the pouch of Douglas. Other systemic examinations were negative. $X$-ray examinations of the abdomen revealed staghorn and multiple calculi in the left kidney, no fluid levels, and no free gas under the diaphragm.

The abdomen was opened through a right middle paramedian incision. There was foul-smelling purulent exudate all over the peritoneal cavity. Pus was oozing through a small hole $(1 \mathrm{~cm}$. in diameter) in the posterior parietal peritoneum just medial to the descending colon. With some difficulty the descending colon was mobilized from the left kidney. The pus was seen to be coming down from a pyonephrosis from the already diagnosed calculus condition of the left kidney. The patient's poor general condition precluded immediate nephrectomy, so a drain was inserted through a stab wound retroperitoneally in the pyonephrotic area. The abdomen was closed in layers. Post-operatively the patient was given 2 pints $(1,140 \mathrm{ml}$.) of blood and ampicillin.

The patient recovered from her general peritonitis without incident and her wound healed. But subsequently the perirenal abscess re-formed. Surgical drainage was carried out and the patient was given sulphadiazine and tetracycline for one week. Recovery thereafter was uneventful.

She was readmitted on 24 September for left nephrectomy, which was carried out on 27 September, and a small shrivelled kidney containing stones was removed. Histology revealed a chronic pyelonephritic kidney with loss of renal tissue and fibrosis.

Her post-operative convalescence was entirely satisfactory until 5 p.m. on 5 October, when severe pain developed suddenly in the right renal area, with anuria. On palpation the right kidney was swollen and tender. Next morning radiography revealed an acutely obstructed remaining kidney. There was no evidence of an opaque calculus. Cystoscopy was carried out and the right ureteric crifice was seen pouting into the bladder, with yellowishwhite matter in the centre of the orifice. A ureteric catheter was passed up the orifice with ease, dislodging the seemingly inspissated bead of mucopus. A copious flow of urine resulted. The ureteric 